\title{
Enhanced insulin sensitivity after acute exercise is not associated with changes in high-molecular weight adiponectin concentration in plasma
}

\author{
Faidon Magkos ${ }^{1,2}$, B Selma Mohammed ${ }^{2}$ and Bettina Mittendorfer ${ }^{2}$ \\ ${ }^{1}$ Department of Nutrition and Dietetics, Harokopio University, 70 El. Venizelou Avenue, Athens 17671, Greece and ${ }^{2}$ Center for Human Nutrition, \\ Washington University School of Medicine, St Louis, Missouri, USA
}

(Correspondence should be addressed to F Magkos at Department of Nutrition and Dietetics, Harokopio University; Email: faimag@hua.gr)

\begin{abstract}
Background and objective: The effect of exercise on the plasma concentration of high-molecular weight (HMW) adiponectin (i.e. the biologically active form of circulating adiponectin) and the possible role of HMW adiponectin in mediating the exercise-induced enhancement of insulin action are not known. The aim of this study was to evaluate the relationship between the post-exercise increase in insulin sensitivity and plasma HMW adiponectin concentration.

Design and methods: We measured total and HMW adiponectin concentrations in plasma using an ELISA kit, and insulin sensitivity using the updated homeostasis model assessment of insulin sensitivity (HOMA2-IS) score in the basal, overnight fasted state, once $\sim 12 \mathrm{~h}$ after a single bout of moderateintensity endurance exercise and once after an equivalent period of rest, in 27 healthy men and women (age: $29 \pm 1$ years and body mass index: $24.7 \pm 0.8 \mathrm{~kg} / \mathrm{m}^{2}$ ).

Results: The HOMA2-IS score was $18 \pm 7 \%$ greater after exercise than after rest $(229 \pm 20$ and 196 \pm 17 respectively; $P=0.006)$, whereas the concentrations of total adiponectin $(7.8 \pm 0.5$ and 7.7 $\pm 0.5 \mathrm{mg} / \mathrm{l}$ respectively; $P=0.597)$ and HMW adiponectin $(3.0 \pm 0.3$ and $3.0 \pm 0.3 \mathrm{mg} / \mathrm{l}$ respectively; $P=0.625)$ were not different. The exercise-induced change in HOMA2-IS score was not related to changes in total and HMW adiponectin concentrations $(P>0.3)$.

Conclusions: Changes in HMW adiponectin concentration are not involved in the acute exerciseinduced enhancement of insulin action.

European Journal of Endocrinology 162 61-66
\end{abstract}

\section{Introduction}

Adiponectin, in particular, the high-molecular weight (HMW) adiponectin isoform (1-4), has potent antiatherogenic, anti-inflammatory, and anti-diabetic effects (5). Decreased plasma adiponectin concentrations are characteristic of insulin-resistant states, and in animals, adiponectin administration or overexpression improves insulin sensitivity $(6,7)$. In human subjects, adiponectin concentrations are reduced in obese, glucose-intolerant, and type 2 diabetic subjects, and weight loss and treatment with thiazolidinediones or rimonabant which improve insulin sensitivity also increase plasma adiponectin concentrations (7-9). Aerobic exercise is well known to enhance insulin action, both acutely, i.e. after a single bout of exercise, and chronically, i.e. after regular exercise training (10-12). However, it is not known whether the exercise-induced change in insulin sensitivity is accompanied by and possibly mediated by changes in adiponectin availability (13). Some studies report an increase, some report no change, and some even report a decrease in total plasma adiponectin concentration after a single session of aerobic exercise or endurance training (14). Part of this discrepancy could be due to the fact that only total plasma adiponectin but not HMW adiponectin concentration was measured in these studies. Furthermore, in studies that evaluated changes in HMW adiponectin concentration after a single bout of aerobic exercise, measurements were made within 30 min of exercise cessation $(15,16)$, whereas the acute exercise-induced enhancement of insulin action is typically not evident until later during recovery (12). Failure to observe changes in HMW adiponectin concentration $(15,16)$ might therefore have been due to improper timing of the measurements.

The purpose of this study was to determine the relationship between the exercise-induced changes in insulin sensitivity and HMW adiponectin concentration in plasma during the late phase of recovery from a single bout of moderate-intensity aerobic exercise. We made our measurements in healthy men and women in the basal state after an overnight fast, once $\sim 12 \mathrm{~h}$ after a single evening bout of endurance exercise and once 
after a time-matched resting trial. Insulin sensitivity was determined using the updated homeostasis model assessment of insulin sensitivity (HOMA2-IS) score, which is based on improved modeling algorithms (17).

\section{Materials and methods}

\section{Subjects and preliminary testing}

Twenty-seven men and women (age: $29 \pm 1$ years; body mass index: $24.7 \pm 0.8 \mathrm{~kg} / \mathrm{m}^{2}$; peak oxygen consumption ( $\mathrm{VO}_{2}$ peak): $39 \pm 2 \mathrm{ml} / \mathrm{kg}$ per min; means \pm s.E.M.) volunteered for the study. All the subjects were considered to be in good health after completing a medical evaluation, which included a history and physical examination and standard blood tests. All were normoglycemic and normolipidemic; none consumed tobacco products or took medications known to affect metabolism. $\mathrm{VO}_{2}$ peak was determined on a bicycle ergometer as described previously (18-20). Written informed consent was obtained from all the subjects before their participation in the study, which was approved by the Human Studies Committee and the General Clinical Research Center (GCRC) Advisory Committee at the Washington University School of Medicine in St Louis, MO, USA.

\section{Experimental protocol}

Each subject completed two time-matched studies within 4 weeks in a randomized order: one after resting and one after cycling on the preceding afternoon. Female subjects performed both trials in the same phase of the menstrual cycle. Subjects were instructed to adhere to their regular diet and to refrain from exercise for a minimum of 3 days before being admitted to the GCRC the afternoon before each study (rest and exercise). For the exercise study, subjects cycled on a semi-recumbent cycle ergometer (EC-C400R Ergometer, Cateye Fitness, Source Distributors, Dallas, TX, USA) for 60-120 min between 1700 and $1900 \mathrm{~h}$. The duration of exercise was variable to bring about a wide range in the exercise-induced changes in insulin sensitivity (21). The workload was set to elicit a $\mathrm{VO}_{2}$ equivalent to $60 \%$ of $\mathrm{VO}_{2}$ peak; $\mathrm{VO}_{2}$ was measured (TrueOne 2400 Metabolic Measurement System, ParvoMedics, Salt Lake City, UT, USA) at regular intervals during exercise, and the workload was adjusted as necessary to maintain the desired $\mathrm{VO}_{2}$ (within $\pm 5 \%$ ). Cardiorespiratory and metabolic measures during exercise are summarized in Table 1. For the resting study, subjects lay on a bed or sat on a chair. After completion of the exercise or the equivalent period of rest, subjects took a shower and then rested on a chair. At $\sim 1930 \mathrm{~h}$, they consumed a standard meal containing $\sim 15 \mathrm{kcal} / \mathrm{kg}$ body weight ( $\sim 55 \%$ of total energy from carbohydrate, $30 \%$ from fat, and 15\% from protein), and then fasted
Table 1 Cardiorespiratory and metabolic measures during the exercise session. Values are means \pm s.E.M.

\begin{tabular}{lc}
\hline Oxygen consumption (ml/kg per min) & $23 \pm 2$ \\
Relative intensity (percent of $\mathrm{VO}_{2}$ peak) & $60 \pm 1$ \\
Respiratory exchange ratio & $0.95 \pm 0.01$ \\
Heart rate (bpm) & $134 \pm 2$ \\
Resistance (watt) & $113 \pm 7$ \\
Net energy expenditure (kcal) & $665 \pm 85$ \\
\hline
\end{tabular}

$\mathrm{VO}_{2}$ peak, peak oxygen consumption. Data represent averages of measurements at 10-30-min intervals during exercise.

(except for water) and rested on a bed until the completion of the study the next day.

At $0700 \mathrm{~h}$ the following morning, an arterialized blood sample was obtained from a heated hand vein for the determination of fasting plasma glucose, insulin, and adiponectin concentrations. Blood was collected in chilled tubes containing heparin (for glucose) or sodium EDTA plus aprotinin (for insulin and adiponectin) and placed immediately on ice. Plasma was separated by centrifugation within 30 min of collection, and samples were stored at $-80{ }^{\circ} \mathrm{C}$ until analysis.

\section{Sample analysis}

Plasma glucose concentration was determined using the glucose oxidase method on an automated glucose analyzer (YSI 2300 STAT PLUS, Yellow Spring Instruments, Yellow Springs, OH, USA). Plasma insulin concentration was measured with a commercially available RIA kit which is specific for insulin (Linco Research, St Louis, MO, USA) (22). Total plasma adiponectin and HMW adiponectin concentrations were determined using a commercially available sandwich ELISA kit (American Laboratory Products Company, Windham, NH, USA), which uses monoclonal antibodies against human adiponectin and protease pretreatment to selectively digest low- and middlemolecular weight adiponectin isoforms $(23,24)$. Insulin sensitivity was assessed with the HOMA2-IS score using the HOMA Calculator v2.2.2 (Diabetes Trials Unit, The Oxford Centre for Diabetes, Endocrinology and Metabolism, Oxford, UK) (17). Validation studies report good correlations $(r=0.7-0.9)$ between HOMA scores and estimates of insulin sensitivity derived from the hyperinsulinemic-euglycemic clamp technique and minimal model analysis (25), and the correlation between plasma adiponectin concentration and HOMA scores $(r=\sim 0.4)$ (26) is as good as the correlation between adiponectin and other measures of insulin sensitivity $(r=0.3-0.6$; hyperinsulinemic-euglycemic clamp, minimal model analysis, and oral glucose tolerance test) (27-29).

\section{Statistical analysis}

Data were analyzed with SPSS v17.0 for Windows (SPSS Inc., Chicago, IL, USA). All data sets were normally distributed according to the Kolmogorov-Smirnov 
Table 2 Glucose, insulin, and adiponectin concentrations and insulin sensitivity after rest and after exercise. Values are means \pm S.E.M.

\begin{tabular}{lccc}
\hline & Rest & Exercise & $\boldsymbol{P}$ value \\
\hline Glucose (mmol/l) & $4.8 \pm 0.1$ & $4.6 \pm 0.1$ & 0.001 \\
Insulin (pmol/l) & $30 \pm 3$ & $27 \pm 3$ & 0.083 \\
HOMA2-IS score & $196 \pm 17$ & $229 \pm 20$ & 0.006 \\
Total adiponectin (mg/l) & $7.7 \pm 0.5$ & $7.8 \pm 0.5$ & 0.597 \\
LMW + MMW adiponectin & $4.7 \pm 0.3$ & $4.8 \pm 0.3$ & 0.189 \\
$\quad$ (mg/l) & & & \\
HMW adiponectin (mg/l) & $3.0 \pm 0.3$ & $3.0 \pm 0.3$ & 0.625 \\
Ratio of HMW-to-total & $0.37 \pm 0.02$ & $0.36 \pm 0.02$ & 0.295 \\
$\quad$ adiponectin & & & \\
\hline
\end{tabular}

HOMA2-IS, updated homeostasis model assessment of insulin sensitivity; LMW + MMW, low- and middle-molecular weight; HMW, high-molecula weight. Data represent averages of duplicate measurements in the basal, overnight fasted state $\sim 12 \mathrm{~h}$ after exercise or an equivalent period of rest.

criteria. Data are presented as means \pm s.E.M. Results after rest and after exercise were compared with Student's paired, two-tailed $t$-test. Relationships between variables of interest were examined with Pearson's correlation analyses. A $P$ value of $<0.05$ was considered statistically significant.

\section{Results}

Plasma glucose concentration was $\sim 5 \%$ lower $(P=0.001)$, plasma insulin concentration was $\sim 10 \%$ lower $(P=0.083)$, and the HOMA2-IS score was $\sim 20 \%$ greater $(P=0.006)$ after exercise than after rest (Table 2). Total plasma adiponectin and HMW adiponectin concentrations and the proportional contribution of HMW adiponectin to total adiponectin concentration (i.e. the ratio of HMW-to-total adiponectin) were not different after rest and after exercise (Table 2). There was no relationship between exercise duration or exercise energy expenditure and the changes in plasma total and HMW adiponectin concentrations (all $P$ values $>0.175$ ).

After the resting trial, the HOMA2-IS score was positively associated with both total $(r=0.641$, $P<0.001)$ and HMW $(r=0.621, P=0.001)$ adiponectin concentrations and the ratio of HMW-to-total adiponectin $(r=0.423, P=0.028)$; however, these relationships were not readily apparent after the exercise trial (all $P$ values $>0.05$ ).

The exercise-induced change in HOMA2-IS was not related to changes in circulating total and HMW adiponectin concentrations or the ratio of HMW-to-total adiponectin (Table 3).

\section{Discussion}

We evaluated the relationship between exercise-induced changes in insulin sensitivity, assessed by the HOMA2-IS score, and total and HMW adiponectin concentrations in the basal, overnight fasted state after a single bout of endurance exercise in healthy men and women. We found that the correlation between total adiponectin concentration and insulin sensitivity observed at rest in our present study and by others (26-29) disappears after exercise. Furthermore, changes in HMW adiponectin concentration (the biologically active isoform of adiponectin) are not involved in increasing insulin sensitivity during the late phase of recovery from a single bout of exercise. Therefore, circulating adiponectin concentration may be an important determinant of insulin action during resting conditions, but it does not mediate the exerciseinduced changes in insulin sensitivity, indicating that other factors are likely to be responsible. The failure of a single bout of exercise to raise total and HMW adiponectin concentrations in plasma is consistent with the results obtained from studies that measured total and HMW adiponectin concentrations immediately (15) or shortly after (16) a single bout of aerobic exercise; however, they are at odds with the results of the studies of endurance training which reportedly raises plasma HMW adiponectin concentration (30, 31). Increased HMW adiponectin availability therefore appears to be an adaptation to chronic exercise, and is not necessary to mediate the increase in insulin action acutely after exercise.

Our findings complement and expand our current knowledge regarding the effect of exercise on adiponectin homeostasis. Although adiponectin secretion from subcutaneous adipose tissue increases significantly during exercise (32), only some but not all the studies

Table 3 Relationship between exercise-induced changes in insulin sensitivity and circulating adiponectin.

\begin{tabular}{lcc}
\hline & $\begin{array}{c}\text { Change in } \\
\text { HOMA2-IS score }\end{array}$ & $\begin{array}{c}\text { Percent change } \\
\text { in HOMA2-IS score }\end{array}$ \\
\hline Change in total adiponectin concentration & $-0.207(0.301)$ & - \\
Percent change in total adiponectin concentration & - & $-0.125(0.534)$ \\
Change in HMW adiponectin concentration & $-0.201(0.314)$ & - \\
Percent change in HMW adiponectin concentration & - & $-0.138(0.492)$ \\
Change in the ratio of HMW-to-total adiponectin & $-0.107(0.596)$ & - \\
Percent change in the ratio of HMW-to-total adiponectin & - & $-0.095(0.636)$
\end{tabular}

Values are Pearson's linear correlation coefficients $(r)$ with $P$ values given in the parentheses. HOMA2-IS, updated homeostasis model assessment of insulin sensitivity; HMW, high-molecular weight. 
report an increase in total adiponectin concentration after a single bout of exercise (14). More importantly, however, only two studies to date have measured the concentration of HMW adiponectin, i.e. the biologically active isoform of adiponectin (1-4), during and/or after exercise and found no changes, even though measurements were made during the early phase of recovery from exercise ( $\leq 30 \mathrm{~min}$ post-exercise) $(15,16)$, whereas the acute exercise-induced enhancement of insulin action is typically not evident until $>3 \mathrm{~h}$ after exercise cessation (12). To our knowledge, acute exercise-induced changes in plasma HMW adiponectin concentration during the prolonged recovery period have never been examined. Actually, only one study has measured total adiponectin concentration up to $48 \mathrm{~h}$ after a single bout of exercise and did not observe any changes, but unfortunately the amount of exercise performed in that study was insufficient to increase insulin sensitivity (33). In our study, insulin sensitivity improved after exercise by $18 \pm 7 \%$, but we found no correlation between the exercise-induced changes in insulin sensitivity and plasma adiponectin concentration. We therefore conclude that the insulinsensitizing effect of a single bout of exercise is not mediated by changes in plasma HMW adiponectin availability.

The mechanisms responsible for the increase in insulin sensitivity after acute exercise are not entirely clear. Depletion of muscle glycogen leads to enhanced insulin-mediated glucose uptake in the previously exercised muscles to facilitate glycogen replenishment (34, 35). Intramuscular triglyceride is also closely associated with insulin sensitivity (36), and the depletion of skeletal muscle lipid stores during exercise in conjunction with enhanced lipid oxidation after exercise could also facilitate muscle insulin action (37). On the other hand, although both chronic (12 months) (38) and acute (48 h) (39) diet-induced energy deficits improve insulin sensitivity, the negative energy balance induced by exercise in our study is unlikely to be the cause for the exercise-induced enhancement of insulin action because a single bout of prolonged endurance exercise brings about an increase in insulin sensitivity the next morning regardless of whether the calories expended during exercise are replaced by overfeeding (zero energy balance) or not (negative energy balance) (40). Likewise, total and HMW adiponectin concentrations increase after prolonged hypocaloric diets (41, 42); however, they are not affected by short-term (4 days of $-800 \mathrm{kcal} /$ day) (43) or acute (48 h of food deprivation) (44) energy deficits.

The acute effect of exercise on plasma adiponectin concentration contrasts that of regular exercise training where increased HMW adiponectin concentrations and improved insulin sensitivity were observed 48-72 h after the last bout of exercise $(30,31)$. The reason for the different response of HMW adiponectin concentration to acute and chronic exercise is not entirely clear, but it could be due to the training-induced decrease in body weight and body fat in these studies $(30,31)$ rather than to an adaptive response to repeated bouts of exercise. Adipose tissue adiponectin gene expression and plasma concentration increase after weight loss $(41,45)$, and training-induced changes in total plasma adiponectin concentration correlate inversely with the corresponding changes in body weight and body fat $(46,47)$. In fact, two recent studies demonstrated that short-term (7 days) endurance (48) and long-term (12 weeks) resistance (49) exercise training do not increase total and HMW adiponectin concentrations in the absence of weight loss.

We used the HOMA score as an index of whole-body insulin sensitivity. Therefore, we cannot determine whether the exercise-induced changes in insulin sensitivity occurred in the muscle or the liver. However, this does not affect the conclusion derived from our study, i.e. that changes in total and HMW adiponectin concentrations are not responsible for the exerciseinduced increase in insulin sensitivity. Furthermore, we only measured the plasma concentration of total and HMW adiponectin, so we do not know whether exercise might have caused an increase in the sensitivity to adiponectin. There is evidence that exercise (acute and chronic) increases adiponectin receptor expression in skeletal muscle $(30,50)$ and adipose tissue (51). Therefore, changes in adiponectin receptor tissue density after exercise, rather than changes in circulating total and HMW adiponectin, may be of physiological importance for the increase in insulin sensitivity after exercise. Unfortunately, assessing sensitivity to adiponectin in vivo is currently not feasible as many factors, both technical and physiological, make administration of the protein rather challenging (52).

In summary, despite increased insulin sensitivity we found no evidence of an exercise-induced change in the concentration of adiponectin (total and HMW) in plasma in the basal, post-absorptive state. These observations argue against the involvement of adiponectin in the acute exercise-induced enhancement of insulin action.

\section{Declaration of interest}

The authors have no conflict of interest that could be perceived as prejudicing the impartiality of the research reported.

\section{Funding}

This study was supported by National Institutes of Health grants AR 49869, HD 057796, DK 56341 (Clinical Nutrition Research Unit), and RR 00954 (Biomedical Mass Spectrometry Resource); grant number UL1 RR024992 from the National Center for Research Resources (NCRR), a component of the National Institutes of Health (NIH) and NIH Roadmap for Medical Research; and grants from the American Heart Association (0365436Z and 0510015Z). 


\section{Author contribution statement}

F Magkos and B Mittendorfer designed the research; F Magkos, B Selma Mohammed, and B Mittendorfer performed the studies; and F Magkos and B Mittendorfer wrote the manuscript.

\section{Acknowledgements}

The authors wish to thank Megan Steward for subject recruitment, the nursing staff of the Clinical Research Center for their technical assistance, and the study subjects for their participation.

\section{References}

1 Pajvani UB, Hawkins M, Combs TP, Rajala MW, Doebber T, Berger JP, Wagner JA, Wu M, Knopps A, Xiang AH, Utzschneider KM, Kahn SE, Olefsky JM, Buchanan TA \& Scherer PE. Complex distribution, not absolute amount of adiponectin, correlates with thiazolidinedione-mediated improvement in insulin sensitivity. Journal of Biological Chemistry 2004 279 12152-12162.

2 Fisher FF, Trujillo ME, Hanif W, Barnett AH, McTernan PG, Scherer PE \& Kumar S. Serum high molecular weight complex of adiponectin correlates better with glucose tolerance than total serum adiponectin in Indo-Asian males. Diabetologia $2005 \mathbf{4 8}$ 1084-1087.

3 Hara K, Horikoshi M, Yamauchi T, Yago H, Miyazaki O, Ebinuma H, Imai Y, Nagai R \& Kadowaki T. Measurement of the high-molecular weight form of adiponectin in plasma is useful for the prediction of insulin resistance and metabolic syndrome. Diabetes Care 200629 1357-1362.

4 von Eynatten M, Lepper PM \& Humpert PM. Total and highmolecular weight adiponectin in relation to metabolic variables at baseline and in response to an exercise treatment program: comparative evaluation of three assays: response to Bluher et al. Diabetes Care 200730 e67-e68.

5 Okamoto Y, Kihara S, Funahashi T, Matsuzawa Y \& Libby P. Adiponectin: a key adipocytokine in metabolic syndrome. Clinical Science $2006110267-278$

6 Kadowaki T, Yamauchi T, Kubota N, Hara K, Ueki K \& Tobe K. Adiponectin and adiponectin receptors in insulin resistance, diabetes, and the metabolic syndrome. Journal of Clinical Investigation 2006116 1784-1792.

7 Kadowaki T \& Yamauchi T. Adiponectin and adiponectin receptors. Endocrine Reviews 200526 439-451.

8 Havel PJ. Update on adipocyte hormones: regulation of energy balance and carbohydrate/lipid metabolism. Diabetes 200453 S143-S151

9 Migrenne S, Lacombe A, Lefevre AL, Pruniaux MP, Guillot E, Galzin AM \& Magnan C. Adiponectin is required to mediate rimonabant-induced improvement of insulin sensitivity but not body weight loss in diet-induced obese mice. American Journal of Physiology. Regulatory, Integrative and Comparative Physiology 2009 296 R929-R935.

10 Borghouts LB \& Keizer HA. Exercise and insulin sensitivity: a review. International Journal of Sports Medicine 200021 1-12.

11 Perseghin G, Price TB, Petersen KF, Roden M, Cline GW, Gerow K, Rothman DL \& Shulman GI. Increased glucose transportphosphorylation and muscle glycogen synthesis after exercise training in insulin-resistant subjects. New England Journal of Medicine 1996335 1357-1362.

12 Magkos F \& Sidossis LS. Exercise and insulin sensitivity. Where do we stand? You'd better run! European Journal of Endocrinology 2009 $422-25$.

13 Kraemer RR \& Castracane VD. Exercise and humoral mediators of peripheral energy balance: ghrelin and adiponectin. Experimental Biology and Medicine 2007232 184-194.
14 Simpson KA \& Singh MA. Effects of exercise on adiponectin: a systematic review. Obesity 200816 241-256.

15 Bobbert T, Wegewitz U, Brechtel L, Freudenberg M, Mai K, Mohlig M, Diederich S, Ristow M, Rochlitz H, Pfeiffer AF \& Spranger J. Adiponectin oligomers in human serum during acute and chronic exercise: relation to lipid metabolism and insulin sensitivity. International Journal of Sports Medicine $2007281-8$.

16 Numao S, Suzuki M, Matsuo T, Nomata Y, Nakata Y \& Tanaka K. Effects of acute aerobic exercise on high-molecular-weight adiponectin. Medicine and Science in Sports and Exercise $2008 \mathbf{4 0}$ 1271-1276.

17 Levy JC, Matthews DR \& Hermans MP. Correct homeostasis model assessment (HOMA) evaluation uses the computer program. Diabetes Care 199821 2191-2192.

18 Magkos F, Patterson BW, Mohammed BS \& Mittendorfer B. A single 1-h bout of evening exercise increases basal FFA flux without affecting VLDL-triglyceride and VLDL-apolipoprotein B-100 kinetics in untrained lean men. American Journal of Physiology. Endocrinology and Metabolism 2007292 E1568-E1574.

19 Magkos F, Patterson BW, Mohammed BS \& Mittendorfer B. Basal adipose tissue and hepatic lipid kinetics are not affected by a single exercise bout of moderate duration and intensity in sedentary women. Clinical Science 2009116 327-334.

20 Magkos F, Wright DC, Patterson BW, Mohammed BS \& Mittendorfer B. Lipid metabolism response to a single, prolonged bout of endurance exercise in healthy young men. American Journal of Physiology. Endocrinology and Metabolism 2006290 E355-E362.

21 Magkos F, Tsekouras Y, Kavouras SA, Mittendorfer B \& Sidossis LS. Improved insulin sensitivity after a single bout of exercise is curvilinearly related to exercise energy expenditure. Clinical Science $200811459-64$.

22 Manley SE, Stratton IM, Clark PM \& Luzio SD. Comparison of 11 human insulin assays: implications for clinical investigation and research. Clinical Chemistry 200753 922-932.

23 Ebinuma H, Miyazaki O, Yago H, Hara K, Yamauchi T \& Kadowaki T. A novel ELISA system for selective measurement of human adiponectin multimers by using proteases. Clinica Chimica Acta 2006372 47-53.

24 Magkos F \& Sidossis LS. Recent advances in the measurement of adiponectin isoform distribution. Current Opinion in Clinical Nutrition and Metabolic Care 200710 571-575.

25 Wallace TM, Levy JC \& Matthews DR. Use and abuse of HOMA modeling. Diabetes Care 200427 1487-1495.

26 Matsubara M, Katayose S \& Maruoka S. Decreased plasma adiponectin concentrations in nondiabetic women with elevated homeostasis model assessment ratios. European Journal of Endocrinology 2003148 343-350.

27 Cnop M, Havel PJ, Utzschneider KM, Carr DB, Sinha MK, Boyko EJ, Retzlaff BM, Knopp RH, Brunzell JD \& Kahn SE. Relationship of adiponectin to body fat distribution, insulin sensitivity and plasma lipoproteins: evidence for independent roles of age and sex. Diabetologia 200346 459-469.

28 Tschritter O, Fritsche A, Thamer C, Haap M, Shirkavand F, Rahe S, Staiger H, Maerker E, Haring H \& Stumvoll M. Plasma adiponectin concentrations predict insulin sensitivity of both glucose and lipid metabolism. Diabetes 200352 239-243.

29 Weyer C, Funahashi T, Tanaka S, Hotta K, Matsuzawa Y, Pratley RE \& Tataranni PA. Hypoadiponectinemia in obesity and type 2 diabetes: close association with insulin resistance and hyperinsulinemia. Journal of Clinical Endocrinology and Metabolism 200186 1930-1935.

30 O'Leary VB, Jorett AE, Marchetti CM, Gonzalez F, Phillips SA, Ciaraldi TP \& Kirwan JP. Enhanced adiponectin multimer ratio and skeletal muscle adiponectin receptor expression following exercise training and diet in older insulin-resistant adults. American Journal of Physiology. Endocrinology and Metabolism 2007293 E421-E427. 
31 Bluher M, Brennan AM, Kelesidis T, Kratzsch J, Fasshauer M, Kralisch S, Williams CJ \& Mantzoros CS. Total and high-molecular weight adiponectin in relation to metabolic variables at baseline and in response to an exercise treatment program: comparative evaluation of three assays. Diabetes Care 200730 280-285.

32 Hojbjerre L, Rosenzweig M, Dela F, Bruun JM \& Stallknecht B. Acute exercise increases adipose tissue interstitial adiponectin concentration in healthy overweight and lean subjects. European Journal of Endocrinology 2007157 613-623.

33 Jamurtas AZ, Theocharis V, Koukoulis G, Stakias N, Fatouros IG, Kouretas D \& Koutedakis Y. The effects of acute exercise on serum adiponectin and resistin levels and their relation to insulin sensitivity in overweight males. European Journal of Applied Physiology 200697 122-126.

34 Annuzzi G, Riccardi G, Capaldo B \& Kaijser L. Increased insulinstimulated glucose uptake by exercised human muscles one day after prolonged physical exercise. European Journal of Clinical Investigation $1991 \mathbf{2 1} 6-12$.

35 Holloszy JO. Exercise-induced increase in muscle insulin sensitivity. Journal of Applied Physiology 200599 338-343.

36 Goodpaster $\mathrm{BH}$ \& Brown NF. Skeletal muscle lipid and its association with insulin resistance: what is the role for exercise? Exercise and Sport Sciences Reviews 200533 150-154.

37 Bruce CR \& Hawley JA. Improvements in insulin resistance with aerobic exercise training: a lipocentric approach. Medicine and Science in Sports and Exercise 200436 1196-1201.

38 Weiss EP, Racette SB, Villareal DT, Fontana L, Steger-May K, Schechtman KB, Klein S \& Holloszy JO. Improvements in glucose tolerance and insulin action induced by increasing energy expenditure or decreasing energy intake: a randomized controlled trial. American Journal of Clinical Nutrition 200684 1033-1042.

39 Kirk E, Reeds DN, Finck BN, Mayurranjan SM, Patterson BW \& Klein S. Dietary fat and carbohydrates differentially alter insulin sensitivity during caloric restriction. Gastroenterology 2009136 $1552-1560$.

40 Fox AK, Kaufman AE \& Horowitz JF. Adding fat calories to meals after exercise does not alter glucose tolerance. Journal of Applied Physiology 200497 11-16.

41 Madsen EL, Rissanen A, Bruun JM, Skogstrand K, Tonstad S, Hougaard DM \& Richelsen B. Weight loss larger than $10 \%$ is needed for general improvement of levels of circulating adiponectin and markers of inflammation in obese subjects: a 3-year weight loss study. European Journal of Endocrinology $2008 \mathbf{1 5 8}$ 179-187.

42 Polak J, Kovacova Z, Jacek M, Klimcakova E, Kovacikova M, Vitkova M, Kuda O, Sebela M, Samcova E \& Stich V. An increase in plasma adiponectin multimeric complexes follows hypocaloric diet-induced weight loss in obese and overweight pre-menopausal women. Clinical Science 2007112 557-565.
43 Imbeault P, Pomerleau M, Harper ME \& Doucet E. Unchanged fasting and postprandial adiponectin levels following a 4-day caloric restriction in young healthy men. Clinical Endocrinology 200460 429-433.

44 Gavrila A, Chan JL, Yiannakouris N, Kontogianni M, Miller LC, Orlova C \& Mantzoros CS. Serum adiponectin levels are inversely associated with overall and central fat distribution but are not directly regulated by acute fasting or leptin administration in humans: cross-sectional and interventional studies. Journal of Clinical Endocrinology and Metabolism 2003 88 4823-4831.

45 Coughlin CC, Finck BN, Eagon JC, Halpin VJ, Magkos F, Mohammed BS \& Klein S. Effect of marked weight loss on adiponectin gene expression and plasma concentrations. Obesity 200715 640-645.

46 Hara T, Fujiwara H, Nakao H, Mimura T, Yoshikawa T \& Fujimoto S. Body composition is related to increase in plasma adiponectin levels rather than training in young obese men. European Journal of Applied Physiology 2005 94 520-526.

47 Marcell TJ, McAuley KA, Traustadottir T \& Reaven PD. Exercise training is not associated with improved levels of C-reactive protein or adiponectin. Metabolism 200554 533-541.

48 Bloem CJ \& Chang AM. Short-term exercise improves beta-cell function and insulin resistance in older people with impaired glucose tolerance. Journal of Clinical Endocrinology and Metabolism 200893 387-392.

49 Ando D, Hosaka Y, Suzuki K \& Yamagata Z. Effects of exercise training on circulating high molecular weight adiponectin and adiponectin oligomer composition: a randomized controlled trial. Journal of Atherosclerosis and Thrombosis, 2009 In press.

50 Bluher M, Bullen JW Jr, Lee JH, Kralisch S, Fasshauer M, Kloting N, Niebauer J, Schon MR, Williams CJ \& Mantzoros CS. Circulating adiponectin and expression of adiponectin receptors in human skeletal muscle: associations with metabolic parameters and insulin resistance and regulation by physical training. Journal of Clinical Endocrinology and Metabolism $2006912310-2316$.

51 Bluher M, Williams CJ, Kloting N, Hsi A, Ruschke K, Oberbach A, Fasshauer M, Berndt J, Schon MR, Wolk A, Stumvoll M \& Mantzoros CS. Gene expression of adiponectin receptors in human visceral and subcutaneous adipose tissue is related to insulin resistance and metabolic parameters and is altered in response to physical training. Diabetes Care 200730 3110-3115.

52 Shetty S, Kusminski CM \& Scherer PE. Adiponectin in health and disease: evaluation of adiponectin-targeted drug development strategies. Trends in Pharmacological Sciences $200930234-239$.

Received 8 October 2009

Accepted 26 October 2009 